\title{
Protein degradation during reconsolidation as a mechanism for memory reorganization
}

\author{
Bong-Kiun Kaang ${ }^{1,2 *}$ and Jun-Hyeok Choi ${ }^{1}$ \\ National Creative Research Initiative Center for Memory, Department of Biological Sciences, College of Natural Sciences, Seoul National University, Seoul, South Korea \\ Department of Brain and Cognitive Sciences, College of Natural Sciences, Seoul National University, Seoul, South Korea
}

Edited by:

Yadin Dudai, The Weizmann Institute of Science, Israel

\section{Reviewed by:}

Kobi Rosenblum, Haifa University, Israel

Todd Sacktor, State University of New York Downstate Medical Center, USA

\section{*Correspondence:}

Bong-Kiun Kaang, Department of

Biological Sciences, Seoul National

University, 599 Gwanangno, Seoul

151-747, Korea.

e-mail:kaang@snu.ac.kr
Memory is a reference formed from a past experience that is used to respond to present situations. However, the world is dynamic and situations change, so it is important to update the memory with new information each time it is reactivated in order to adjust the response in the future. Recent researches indicate that memory may undergo a dynamic process that could work as an updating mechanism. This process which is called reconsolidation involves destabilization of the memory after it is reactivated, followed by restabilization. Recently, it has been demonstrated that the initial destabilization process of reconsolidation requires protein degradation. Using protein degradation inhibition as a method to block reconsolidation, recent researches suggest that reconsolidation, especially the protein degradation-dependent destabilization process is necessary for memory reorganization.

Keywords: protein degradation, reconsolidation, memory reorganization, updating mechanism

\section{INTRODUCTION}

Memory can be stored for either a relatively short or a long period of time. For a memory to be stored long-term, it has to be "consolidated." Through the protein synthesis-dependent consolidation process, the information is stabilized as a long-term memory that is relatively insensitive to disruption. Maintaining a memory, without retrieval, does not critically involve transient protein synthesis. After it is retrieved, however, it requires protein synthesis within a specific period, in order to recover the memory from a labile state. Without proper transient protein synthesis, the memory seems to be impaired, suggesting that protein synthesis is required to recover the memory from a certain state where the consolidated memory is destabilized. This post-retrieval, protein-dependent process is termed reconsolidation (Misanin et al., 1968; Nader et al., 2000). The early studies on reconsolidation focused on the consolidationlike restabilization process. In this article, however, we will focus on recent studies exploring the mechanism of destabilization induced by reactivation of a previously consolidated memory and show how these findings can be used to demonstrate that reconsolidation can work as an updating mechanism. We will also discuss on the physiological role of this process for memory updating and reorganization.

\section{RETRIEVAL-INDUCED DESTABILIZATION}

The post-retrieval destabilization process of reconsolidation may involve active loss of components maintaining the memory. Lee et al. (2008) have demonstrated that this active destabilization process requires ubiquitin-proteasome-dependent protein degradation. Inhibition of proteasome activity in the hippocampus after retrieval of contextual fear memory prevented becoming labile, possibly by blocking the destabilization process. Proteasome inhibitor treated together with protein synthesis inhibitor prevented the amnesic effect of protein synthesis inhibitor, preserving the fear memory. On the other hand, proteasome inhibitor itself had no effect on either reconsolidation or consolidation of contextual fear memory.
Polyubiquitinated protein level in the crude synaptosomal fraction has increased after retrieval of contextual fear memory. As the proteasome complex recognizes their target by the polyubiquitin, polyubiquitinated protein level indicates the extent of proteasome-dependent protein degradation. In addition, one of the polyubiquitinated synaptic proteins Shank is shown to be decreased through proteasome-dependent degradation after retrieval, reaching the lowest level $2 \mathrm{~h}$ after retrieval. The protein level is recovered to basal state $6 \mathrm{~h}$ after retrieval. Retrieval-induced protein degradation seems to be regulated in a target specific manner, as polyubiquitination of Shank and Guanylate kinase-associated protein (GKAP) increased after retrieval, while a postsynaptic density protein PSD-95 was not. Shank and GKAP are known to be regulated by synaptic activity in primary neuron culture (Ehlers, 2003; Hung et al., 2010), which is in good agreement with the destabilization process. These results suggest that specific synaptic proteins are destabilized after retrieval through the ubiquitin-proteasomedependent degradation pathway. The fact that PSD-95, a scaffolding protein associated to receptors is not degraded while master scaffolding proteins Shank and GKAP are degraded may give an insight about how the master scaffolding structure is destabilized while the receptor complex itself is preserved, at least in terms of protein degradation. Although an important scaffolding protein PSD-95 may be preserved, the total scaffolding structure is at least partly destabilized, which is reconstructed afterward. There is still a possibility that proximal structure of the receptor complex may also be disassembled by other mechanisms.

There is evidence that NMDA receptor, L-type voltage gated calcium channel (LVGCC), and cannabinoid receptor 1 (CB1 receptor) are also required for retrieval-induced destabilization. NMDA receptor antagonist treated locally in amygdala prevented the amnesic effect of anisomycin in cued fear memory (Ben Mamou et al., 2006). Inhibitor of LVGCC and CB1 receptor treated either systemically or locally in hippocampus blocked the amnesic effect of anisomycin in contextual fear memory (Suzuki et al., 
2008). Considering the nature of these molecules as channels and receptors, these might work as upstream molecules of retrievalinduced protein degradation. Interestingly, CaMKII which can be activated by NMDA receptor and LVGCC are known to phosphorylate a subunit of proteasome complex, which leads to increased activity (Djakovic et al., 2009). CaMKII $\alpha$ is also known to work as a scaffold protein that translocate proteasome from the dendritic shaft to the synaptic spines induced by synaptic activity in primary neuron culture (Bingol et al., 2010). These two researches support the hypothesis that NMDA receptor and LVGCC are activated at the initial stage of retrieval-induced destabilization, regulating protein degradation. However, more researches are required to directly demonstrate the linkage between these molecules in destabilization in vivo.

\section{UPDATING INFORMATION INDUCES RECONSOLIDATION}

As reconsolidation is a dynamic process induced by reactivation of the memory, it has been hypothesized that this might work as an updating mechanism for the reactivated memory (Dudai and Eisenberg, 2004). Related to this idea, there is a body of evidence indicating that reconsolidation occurs only when there is updating information.

\section{TASTE ASSOCIATED MEMORY}

Efforts to demonstrate that reconsolidation works as an updating mechanism first produced numerous reports showing that reconsolidation is induced by updating information. Intra-cortical blockade of protein synthesis in the insular cortex after retrieval of taste-recognition memory disrupted memory only when there was updating information (Rodriguez-Ortiz et al., 2005). Animals have an innate aversion to a novel taste, resulting in reduced consumption. However, when the novel taste is repeatedly exposed, the animal shows a gradual increment of consumption that eventually reaches a plateau. During this period of gradual increase in consumption, intra-cortical anisomycin treatment in the insular cortex after exposure to the taste impairs the memory, not only blocking the incremental increase, but also impairing the previous consumption level. However, when the consumption reaches a plateau, likely when there is no more updating information, animals were tolerant to anisomycin treatment. Another type of taste memory called conditioned taste aversion, where a novel taste is associated with an aversive stimulus, was also impaired by simultaneous anisomycin treatment in the insular cortex and central amygdala after additional conditioned taste aversion trials, while there was no effect after the aversion reached the asymptote where there is no more updating information (Garcia-DeLaTorre et al., 2009).

\section{SPATIAL MEMORY}

In the Morris water maze, a spatial memory task where the animal has to learn the position of a hidden platform in a water filled maze in order to escape from the water, anisomycin treatment during a reminder trial of intermediately trained animals led to impairment in the probe test, while there was no effect in well-trained animals (Rodriguez-Ortiz et al., 2008). A modified version of the water maze, where the platform position is changed day-to-day and new information is acquired every day, was performed by another group (Morris et al., 2006). Intra-hippocampal anisomycin treatment after a reactivation trial following 6 days of training impaired the next day's performance, while anisomycin treatment after reactivation of the normal water maze when the animal was fully trained did not produce an effect.

\section{OBJECT RECOGNITION MEMORY}

Object recognition memory is a memory for object identity. After exposure to two objects, one of the objects is changed to a novel one. If the animal remembers the first two objects, it can discriminate those from the novel object. Intra-hippocampal treatment with anisomycin after reactivating the memory by presenting the initial two objects again did not yield any effect. However, treatment after reactivating the memory by presenting one of the initial objects together with a novel object led to impairment of memory for the reactivated initial object (Rossato et al., 2007). This means that object recognition memory undergoes reconsolidation only when the familiar object is presented together with a novel object. Another group has shown that under conditions where presenting the initial objects does not induce reconsolidation, presenting those same objects with salient, novel contextual information induced reconsolidation of the memory (Winters et al., 2009).

Although these studies show a correlation in which reconsolidation occurs specifically and exclusively when there is updating information under certain conditions, they do not directly demonstrate that reconsolidation is required to update a previously formed memory.

\section{RECONSOLIDATION IS REQUIRED FOR MEMORY REORGANIZATION}

In order to demonstrate that reconsolidation is required to update a previously formed memory, we have to show that blocking reconsolidation prevents incorporation of the updating information. However, many treatments that block reconsolidation are also known to block consolidation, thus making it hard to discriminate whether the failure to incorporate updating information is due to the treatment effect on reconsolidation of the previously formed memory or on consolidation of the new information. Additionally, even a treatment that impair reconsolidation but not consolidation has limitation when it works on the restabilization step, as this would leave the initial memory to be destabilized. With the initial memory destabilized, the incorporation of the updating information would fail even if this process does not directly require reconsolidation. However, treatments that block the destabilization step of reconsolidation may be a good method to demonstrate the requirement of reconsolidation for memory updating, as reconsolidation is blocked while the initial memory itself would be intact.

Using proteasome inhibitor to block reconsolidation, a recent research has demonstrated that retrieval-induced destabilization of the previously formed memory is required to further strengthen the memory (Lee, 2008). After the first contextual fear conditioning, additional conditioning at the same context the next day further strengthen the fear response. The author showed that the strengthening mechanism resembles reconsolidation rather than consolidation, by inhibiting distinct molecular requirements for consolidation and reconsolidation. Then, the author showed that proteasome inhibitor locally treated in hippocampus after the 
second conditioning impaired further strengthening of the memory, leaving memory strength unchanged from the initial memory. Active weakening of the memory, or extinction, has also been demonstrated to be impaired by proteasome inhibitor (Lee et al., 2008). Local treatment of proteasome inhibitor in hippocampus after contextual fear memory extinction trials impaired the decrement of fear response, leaving the fear response level similar to the initial level. There is also evidence that proteasome activity is required for partially modifying the content of the memory rather than modifying the quantitative strength of the memory (Choi et al., 2010). Using an object-place associative memory, where positional memory for four objects is formed and two of the objects are interswitched the next day in order to partially modify the memory, the authors demonstrated that proteasome inhibition treated locally in hippocampus after the second day exposure impaired successful modification of the memory.

These researches demonstrate not only that reconsolidation is required for incorporating updating information to the previously formed memory, but also show that retrieval-induced destabilization is required to reorganize the reactivated memory. Memory strengthening and memory weakening, as well as typical reconsolidation where the memory is recovered to a similar level as the original memory, seem to be initiated by destabilization of the initial memory. These facts give insight for a model of memory reorganization. When the memory is reactivated, it first undergoes a protein degradation-dependent destabilization process. The next step depends on the context of reactivation. When the memory is no longer valid, the destabilized memory either stays in the destabilized state or actively encodes the extinction information. When the memory is further reinforced by additional training, the destabilized memory is restabilized to a stronger state than the initial memory. When the reactivating context is not effective to either weaken or strengthen the initial memory, the destabilized memory is restabilized to a similar state as the initial memory. This alternative destination of the memory after reactivation may also agree with the "dominance of the trace" theory that a certain trace conflicting with another trace (i.e., the "excitatory" original CS-US trace, and an "inhibitory" or new CS-no US) gains control over behavior after the retrieval, which also shows transient sensitivity to consolidation blocker. The restabilization process after the destabilization process is protein synthesis-dependent and could resemble the consolidation process in many aspects.

\section{REFERENCES}

Artinian,J.,McGauran,A.M., De Jaeger,X., Mouledous, L., Frances, B., and Roullet, P. (2008). Protein degradation, as with protein synthesis, is required during not only long-term spatial memory consolidation but also reconsolidation. Eur. J. Neurosci. 27, 3009-3019.

Ben Mamou, C., Gamache, K., and Nader, K. (2006). NMDA receptors are critical for unleashing consolidated auditory fear memories. Nat. Neurosci. 9, 1237-1239.

Bingol, B., Wang, C. F., Arnott, D., Cheng, D., Peng, J., and Sheng, M. (2010).

Autophosphorylated CaMKIIalpha

Choi, J. H., Kim, J. E., and Kaang, B. K.

\section{CONCLUSION AND FUTURE DIRECTIONS}

Reconsolidation has been hypothesized to be an updating mechanism that incorporates the new information to the previously formed memory. However, it was not possible to directly demonstrate that reconsolidation is required to update the memory. Recent studies focusing on the destabilization process of reconsolidation not only has provided understanding of the mechanism of reconsolidation, but also gave a breakthrough to demonstrate the requirement of reconsolidation for memory updating. It also provided insights for the model of memory reorganization, including memory weakening, maintaining, and strengthening after it is reactivated.

There is evidence showing that UPS plays an important role in in vitro model of learning and memory, such as long-term potentiation (LTP) and long-term depression (LTD; Colledge et al., 2003; Fonseca et al., 2006; Hou et al., 2006; Karpova et al., 2006; Deng and Lei, 2007; Cai et al., 2010). Whereas protein degradation did not show a critical role in the consolidation of contextual fear memory, another study showed that protein degradation is required for both consolidation and reconsolidation of spatial memory (Artinian et al., 2008). In contrast with the absolute requirement of protein synthesis for both long-term synaptic plasticity and memory, the involvement of protein degradation may differ between memory types and experimental conditions. There is also a possibility that protein degradation plays more critical role in specific situations, such as in destabilization of the reactivated memory than in general learning and memory process.

Although it is shown that protein degradation is required for the destabilization of reactivated memory, more studies are required to fully understand the molecular mechanism of the destabilization process, especially upstream pathway that activates and regulates protein degradation and downstream target proteins in the preand postsynaptic area. A variety of molecular and cell biological approaches will be helpful to this end and may overcome certain limitations caused possibly by the complex effects of proteosome inhibitors. In addition, it is important to apply these findings to different types of memory, and also to varying situations of memory reorganization.

\section{ACKNOWLEDGMENTS}

This work was supported by the National Creative Research Initiative Program and WCU Program of the Korean Ministry of Science and Technology. Jun-Hyeok Choi is supported by the BK21 fellowship.

pre-existing object-location memory. Mol. Brain 3, 1. acts as a scaffold to recruit proteasomes to dendritic spines. Cell 140, 567-578.

Cai, F., Frey, J. U., Sanna, P.P., and Behnisch, T. (2010). Protein degradation by the proteasome is required for synaptic tagging and the heterosynaptic stabilization of hippocampal late-phase long-term potentiation. Neuroscience 169, 1520-1526. (2010). Protein synthesis and degradation are required for the incorporation of modified information into the
Colledge, M., Snyder, E. M., Crozier, R. A., Soderling, J. A., Jin, Y., Langeberg, L. K., Lu, H., Bear, M. F., and Scott, J. D. (2003). Ubiquitination regulates PSD-95 degradation and AMPA receptor surface expression. Neuron 40, 595-607.

Deng, P. Y., and Lei, S. (2007). Long-term depression in identified stellate neurons of juvenile rat entorhinal cortex. J. Neurophysiol. 97, 727-737.

Djakovic, S. N., Schwarz, L.A., Barylko, B., DeMartino, G. N., and Patrick, G. N.
(2009). Regulation of the proteasome by neuronal activity and calcium/ calmodulin-dependent protein kinase II. J. Biol. Chem. 284, 26655-26665.

Dudai, Y., and Eisenberg, M. (2004). Rites of passage of the engram: reconsolidation and the lingering consolidation hypothesis. Neuron 44, 93-100.

Ehlers, M. D. (2003). Activity level controls postsynaptic composition and signaling via the ubiquitin-proteasome system. Nat. Neurosci. 6, 231-242.

Fonseca, R., Vabulas, R. M., Hartl, F. U., Bonhoeffer, T., and Nagerl, U. V. (2006). A balance of protein synthesis 
and proteasome-dependent degradation determines the maintenance of LTP. Neuron 52, 239-245.

Garcia-DeLaTorre, P., Rodriguez-Ortiz, C. J., Arreguin-Martinez, J. L., CruzCastaneda, P., and Bermudez-Rattoni, F. (2009). Simultaneous but not independent anisomycin infusions in insular cortex and amygdala hinder stabilization of taste memory when updated. Learn. Mem. 16, 514-519.

Hou, L., Antion, M. D., Hu, D., Spencer, C. M., Paylor, R., and Klann, E. (2006). Dynamic translational and proteasomal regulation of fragile $\mathrm{X}$ mental retardation protein controls mGluRdependent long-term depression. Neuron 51, 441-454.

Hung, A. Y., Sung, C. C., Brito, I. L., and Sheng, M. (2010). Degradation of postsynaptic scaffold GKAP and regulation of dendritic spine morphology by the TRIM3 ubiquitin ligase in rat hippocampal neurons. PLoS ONE 5, e9842. doi: 10.1371/journal.pone.0009842

Karpova, A., Mikhaylova, M., Thomas, U., Knopfel, T., and Behnisch, T. (2006). Involvement of protein synthesis and degradation in long-term potentiation of Schaffer collateral CA1 synapses. J. Neurosci. 26, 4949-4955.

Lee, J. L. (2008). Memory reconsolidation mediates the strengthening of memories by additional learning. Nat . Neurosci. 11, 1264-1266.

Lee, S. H., Choi, J. H., Lee, N., Lee, H. R., Kim, J. I., Yu, N. K., Choi, S. L., Kim, H., and Kaang, B. K. (2008). Synaptic protein degradation underlies destabilization of retrieved fear memory. Science 319, 1253-1256.

Misanin, J. R., Miller, R. R., and Lewis, D. J. (1968). Retrograde amnesia produced by electroconvulsive shock after reactivation of a consolidated memory trace. Science 160, 554-555.

Morris, R. G., Inglis, J., Ainge, J. A., Olverman, H. J., Tulloch, J., Dudai, Y., and Kelly, P. A. (2006). Memory reconsolidation: sensitivity of spatial memory to inhibition of protein synthesis in dorsal hippocampus during encoding and retrieval. Neuron 50, 479-489.

Nader, K., Schafe, G. E., and Le Doux, J. E. (2000). Fear memories require protein synthesis in the amygdala for reconsolidation after retrieval. Nature 406, 722-726.
Rodriguez-Ortiz, C. J., De la Cruz, V., Gutierrez, R., and Bermudez-Rattoni, F. (2005). Protein synthesis underlies post-retrieval memory consolidation to a restricted degree only when updated information is obtained. Learn. Mem. 12, 533-537.

Rodriguez-Ortiz, C. J., Garcia-DeLaTorre, P., Benavidez, E., Ballesteros, M. A. and Bermudez-Rattoni, F. (2008). Intrahippocampal anisomycin infusions disrupt previously consolidated spatial memory only when memory is updated. Neurobiol. Learn. Mem. $89,352-359$.

Rossato, J. I., Bevilaqua, L. R., Myskiw, J. C., Medina, J. H., Izquierdo, I., and Cammarota, M. (2007). On the role of hippocampal protein synthesis in the consolidation and reconsolidation of object recognition memory. Learn. Mem. 14, 36-46.

Suzuki, A., Mukawa, T., Tsukagoshi, A., Frankland, P. W., and Kida, S. (2008). Activation of LVGCCs and $\mathrm{CB} 1$ receptors required for destabilization of reactivated contextual fear memories. Learn. Mem. 15, 426-433.
Winters, B. D., Tucci, M. C., and DaCostaFurtado,M. (2009). Older and stronger object memories are selectively destabilized by reactivation in the presence of new information. Learn. Mem. 16, 545-553.

Conflict of Interest Statement: The authors declare that the research was conducted in the absence of any commercial or financial relationships that could be construed as a potential conflict of interest.

Received: 18 May 2010; accepted: 17 January 2011; published online: 01 February 2011.

Citation: Kaang B-K and Choi J-H (2011) Protein degradation during reconsolidation as a mechanism for memory reorganization. Front. Behav. Neurosci. 5:2. doi: 10.3389/ fnbeh.2011.00002

Copyright (C) 2011 Kaang and Choi. This is an open-access article subject to an exclusive license agreement between the authors and Frontiers Media SA, which permits unrestricted use, distribution, and reproduction in any medium, provided the original authors and source are credited. 